\title{
Radio Observations of Supernova Remnants and the Surrounding Interstellar Medium
}

\author{
Gloria Dubner
}

IAFE, Casilla de Correo 67, Suc. 28, 1428 Buenos Aires, Argentina

\begin{abstract}
Supernova Remnants (SNRs) play essential roles in the dynamics of galaxies by injecting large amounts of energy which can accelerate particles generating cosmic radiation, create large, hot, low density bubbles, and maintain turbulent cloud motions. SNRs shock waves can also compress nearby dense clouds, inducing fragmentation and perhaps collapse into protostars, thus joining the extremes in the life-cycle of stars. The present review illustrates how high quality low frequencies radio observations of galactic SNRs and the surrounding interstellar medium (ISM), can help in the understanding of the many aspects of the nature of the remnants, as well as the consequences of their interaction with the ambient gas.
\end{abstract}

\section{Introduction}

A supernova explosion represents the sudden injection of $\sim 10^{51}$ ergs and $\sim 10$ $\mathrm{M}_{\odot}$ of stellar material in a virtually point region of space. A supersonic shock wave is then formed, which expands into the surrounding medium and sweeps up the interstellar gas. The result of the interaction of the stellar ejecta and the blast wave with the ambient gas is known as a supernova remnant (SNR). Depending on the progenitor star, the remnants may include a spinning neutron star or even a black hole.

The physical characteristics of the interaction between the SN shock and the surrounding gas change with time, passing through several distinct stages classically known as "free expansion", "adiabatic blast wave" , "radiative snowplow", and "dispersal" (Woltjer 1972, Chevalier 1977). However, as pointed out by Jones et al. (1998), this simple chronological model may be inadequate to describe the real dynamics of a SNR, and the different phases may be brief, may not occur at all, or may be taking place simultaneously in different portions of the same remnant. In this dynamical complexity, the environments have a crucial role.

Because SNRs involve many different phenomena, the research of SNRs can lead to insights into several problems in astrophysics. To mention only a few, through the multiwavelength study of SNRs, we can learn about: a) the nature of the precursor stars, which together with the physics of the explosion itself, provide key information for the more general study of stellar evolution; b) the central compact sources (neutron stars, black holes) and their coupling with the surrounding plasma; c) shock waves with very high Mach numbers and 
magnetohydrodynamics instabilities at conditions vastly different from those achievable in terrestrial laboratories; and $d$ ) particle acceleration and cosmic radiation origin up to at least $10^{14} \mathrm{eV}$ (Berezhko 1996). Also, by illuminating the nearby environment, SNRs are useful probes of the various phases of the ISM, and provide valuable information about the chemical processes induced by shock waves on surrounding interstellar clouds.

To correctly identify the physical processes and understand the variety of phenomena involved in the evolution of SNRs, the best tools are sensitive radio interferometric images obtained at various wavelengths, (complete over all spatial scales), together with observations in other spectral domains, and surveys of the gaseous surroundings using atomic and molecular lines. The present communication illustrates the contributions to the knowledge of individual sources and to the global comprehension of SNRs based on observations at low radio frequencies of galactic SNRs and the surrounding interstellar medium (ISM).

\section{Radio Continuum Observations of SNRs}

High quality radio continuum observations of SNRs at different frequencies provide information about three main topics: morphology, polarization and spectral index. Ideally, from the study of the morphology, shock structures can be identified and quantified, and the location of contact discontinuities in the fluid can be traced. From polarization studies the intensity and degree of order and orientation of the magnetic fields can be determined. Finally, through the study of spectral indices the energy. spectrum of the accelerated particles can be ascertained. In some good cases most of these goals can be achieved.

Tycho's SNR is a good example where many physical processes have been investigated based on high resolution and high sensitivity observations. The study of this SNR carried out with all four configurations of the VLA in two different epochs has allowed to accurately trace the location of the shock front and the contact discontinuity (Figure 1 left) (Reynoso et al. 1997) and to show the cellular pattern of the magnetic field (Dickel, van Breugel \& Strom 1991, Reynoso et al. 1997). In addition, the expansion of the shock front over a 10 years interval (Figure 1 right) was accurately determined (Reynoso et al. 1997). Significant local variations in the expansion parameter were found, suggesting that more than one dynamic phase can coexist. Based on these very detailed observations, it was also possible to identify and characterize the hydrodynamic instabilities developed along the contact discontinuity (Velázquez et al. 1998) and discover the presence of a compact interstellar cloud that may delay the expansion of the SN shock towards the NE (Reynoso et al. 1999).

Also, the careful VLA imaging at $1.4 \mathrm{GHz}$ of the SNR W50, which contains the powerful source of relativistic jets SS433 in its center, has revealed many faint structures in the synchrotron emission. These features have unambiguously proved the connection between the central microquasar and the surrounding nebula (Dubner et al. 1998). Figure 2 shows the comparison of the radio nebula with the X-ray emission, as obtained with ROSAT by Brinkmann, Aschenbach \& Kawai (1996). The study of the emission at various spectral ranges is not only useful to trace material with different physical conditions and perform plasma diagnostics, but also to understand the energy injection from the central compact 

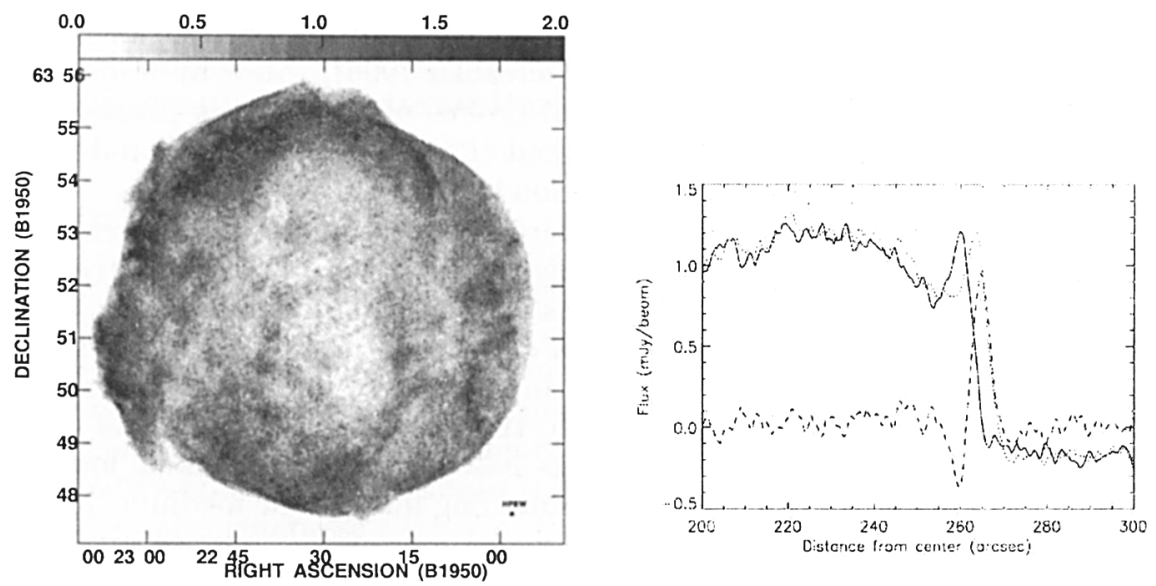

Figure 1. Left: VLA image of Tycho's SNR. Note the well defined wavy-like structures behind the shock front towards the NE, originated in plasma instabilities caused by the presence of a denser cloud in the shock path. Right: Radial profiles showing the two successive positions of the shock front (average of the azimuthal sector between $24^{\circ}$ and $28^{\circ}$ ). The solid line corresponds to epoch 1 and the dotted line corresponds to epoch 2, 10 years later (dashed line is the difference) (from Reynoso et al. 1997)

source. The detailed investigation of a galactic source with these characteristics is an excellent point of reference for the investigation of jets in radio galaxies and QSOs.

In general, however, the information that we can obtain from the radio observations is limited by several factors. For instance, in polarization studies, unknown processes can largely disorder the direction of B fields, and Faraday rotation cannot always be well quantified. Thus, it is not straightforward to image the intensity and orientation of the magnetic field from measurements of polarized intensity and electric field orientation. Also the difficulties in dealing with spectral indices estimates are well known (See D. Green 2000, in these proceedings). Background and zero level corrections at different frequencies and contamination with localized thermal emission limit the accuracy of the results.

The most complete and unbiased information that can be achieved from radio continuum observations is the morphology, even with some unavoidable limitations. The shape of a SNR is a consequence of the dynamical evolution of the shock wave, while the brightness distribution is determined by the state of the plasma inside the SNR. The final appearance of the SNR is the result of three different concurrent factors: the progenitor, the explosion and the ambient medium. The lack of sphericity and uniformity is usually explained by invoking different effects, such as anisotropic bi-polar explosion, non-uniform density distribution of the circumstellar medium (CSM) and ISM, large-scale magnetic 


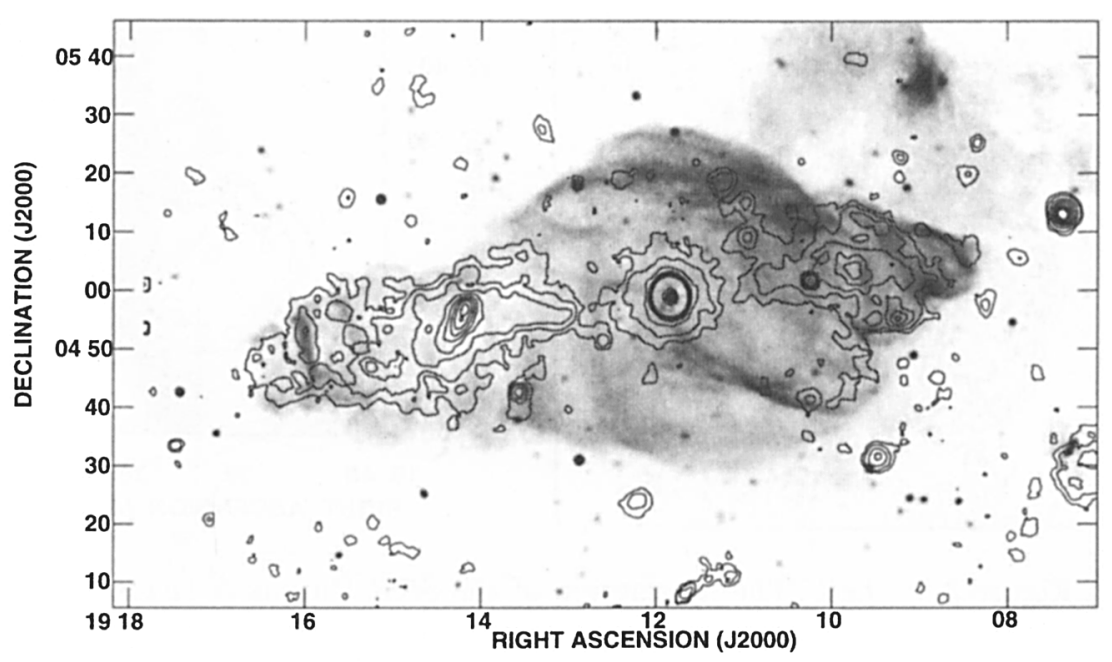

Figure 2. Comparison of the $1.4 \mathrm{GHz}$ VLA image of W50 (greys, from Dubner et al. 1998) with the ROSAT X-ray data (contours, from Brinkmann et al. 1996)

fields effects, the presence of compact sources injecting energy or accelerated particles, etc.

Since the observations provide necessary constraints to the theoretical models, it is important to keep in mind that when we discuss morphology in the synchrotron emission of a SNR, we refer to the bidimensional visual appearance of a 3-dimensional object. Hnatyk \& Petruk (1999) have developed models of non-spherical adiabatic SNRs evolving in ISM with large-scale density gradients (exponential and power-law distributions) and conclude that even a visible spherical shape does not guarantee the uniformity of the surrounding medium and/or isotropy in the explosion. Essentially, the observed morphology of SNRs depends on their orientation with respect to the line of sight and projection effects. Besides, the apparent center of the SNRs may differ from the real progenitor position. As pointed out by Hnatyk \& Petruk (1999), this fact may be important for localizing possible compact stellar remnants in the interior of SNRs.

\section{The Interaction with the Surrounding Medium}

The mutual interaction between the expanding blast wave and the ISM has a remarkable impact both on the expanding remnant and the surrounding gas. The study of the surroundings of SNRs is essential to detect external causes for peculiar morphologies, and discriminate from intrinsic origins. Therefore, in order to obtain a global knowledge of SNRs, it is important to characterize the gaseous surroundings of a number of remnants with different appearances. 

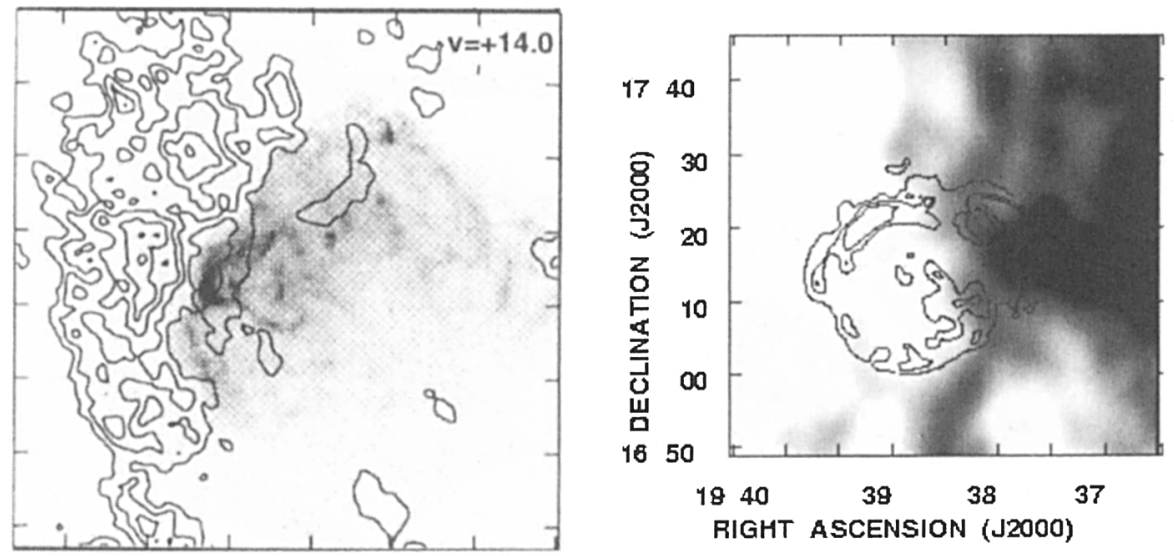

Figure 3. Left: The interaction of the SNR Puppis A (Einstein soft $\mathrm{X}$-rays image shown in grey, as taken from Petre et al. 1982), with the surrounding HI (VLA data in contours, as taken from Reynoso et al. 1995). Right: The SNR 3C400.2 (VLA data, in contours) is evolving close to the border of an extended HI cloud (DRAO data shown in grey). The SNR is asymmetrical, with the larger size to the east, where the SNR expands into a lower density cavity (from Dubner et al. 1994 and Giacani et al. 1998).

Non-uniform environments produce either deceleration of the shock front, when it penetrates a medium with increasing or slowly decreasing density, or acceleration, when the external density decreases fast enough. Of particular interest are the transition zones, where the external density changes abruptly. Figure 3 illustrates two examples of the influence of the inhomogeneous surroundings on the shape of SNRs. The SNR Puppis A (left) encountered a dense cloud towards the east, that appears to have slowed down its expansion and produced a flattened appearance accompanied by enhanced X-ray emission (Dubner et al. 1991, Reynoso et al. 1995). On the contrary, the SNR 3C400.2 (right), encountered a lower density cavity towards the east, where it expanded faster, thus acquiring a "break-out" morphology (Dubner et al. 1994, Giacani et al. 1998).

In some cases, it is the SNR which modifies its environs. Figure 4 shows the case for SS433/W50, a dramatic example of the impact of a SNR on the surrounding gas, where about $2 \times 10^{51}$ ergs of kinetic energy were injected into the ambient medium.

\section{Concluding Remarks}

From the theoretical point of view, considerable progress has been made from the ideal description of a spherical remnant expanding into a uniform ISM. It is now clear that each SNR is the unique product of the history of its progenitor and the influence of its neighbourhood. Also, it has now been realized that the evolutionary paths of SNRs are far from simple. In order to establish global 


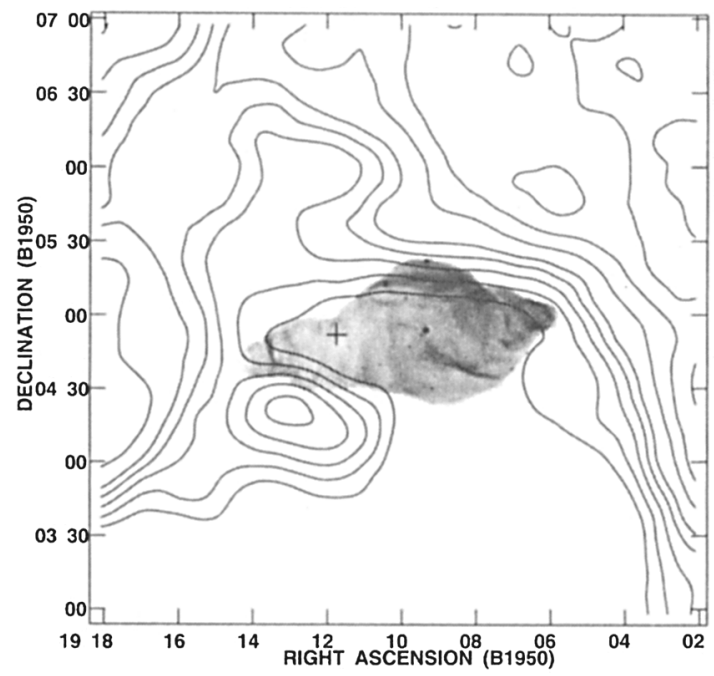

Figure 4. The SNR W50 at $1.4 \mathrm{GHz}$ (VLA image in greys) is pushing away the surrounding HI (contours from NRAO $43 \mathrm{~m}$ telescope data) (Dubner et al. 1999)

models, the theories need to rely on a large observational sample of SNRs of different ages expanding in different environments.

In this context, sensitive and detailed observations at low radio frequencies of the synchrotron emission associated with SNRs and HI studies of the surroundings, are exceptionally helpful. In addition, these new data constitute the necessary starting point for high resolution investigations in other spectral domains, e.g. submillimetric searches for molecular emission in the surrounding medium.

The new generation of interferometers operating at low frequencies will undoubtly provide innovative answers to the many questions that these fascinating objects pose.

Acknowledgments. I am very grateful to the SOC of the Symposium and to the IAU for the travel grant awarded to participate in the Symposium. I thank to W. Brinkmann for kindly providing the ROSAT image of W50 in FITS format. I acknowledge F. Mirabel and W.M.Goss for reading this manuscript. Most of the research summarized in this work was funded by Cooperative Science Programs between NSF and CONICET (Argentina) and through the CONICET grant 4203/96. G.D. is a member of the Carrera del Investigador Científico de CONICET (Argentina).

\section{References}

Berezhko,E.G. 1996, Astropart. Phys., 5, 367

Brinkmann,W., Aschenbach,B., Kawai,N. 1996, A\&A, 312, 306

Chevalier,R.A. 1977, ARA\&A, 15, 175 
Dickel, J. R., van Breugel, W. J. M., \& Strom, R. G. 1991, AJ, 101, 2151

Dubner,G., Winkler,P., Braun,R., Goss,W.M. 1991, AJ, 101, 1466

Dubner,G., Giacani,E., Goss,W. M., Winkler,P. 1994, AJ, 108, 207

Dubner,G., Holdaway,M., Goss,W.M., Mirabel,F. 1998, AJ, 116, 1842

Dubner,G., Giacani,E., Reynoso,E., Goss,W.M., Roth,M., \& Green,A. 1999 AJ, 118,930

Giacani,E., Dubner,G., Cappa,C., Testori,J. 1998, A\&AS133, 61

Jones,T.W., Rudnick,L., Jun,B.-I., Borkowski,K.J., Dubner,G., Frail,D.A., Kank,H., Kassim,N.E., \& Mc Cray,R. 1998, PASP, 110, 125

Hnatyk,B. \& Petruk,O. 1999, A\&A, 344, 295

Petre,R., Canizares,C., Kriss,G., Winkler,P. 1982, ApJ, 258, 22

Reynoso,E., Dubner,G., Goss,W.M., \& Arnal,M. 1995,AJ, 110, 318

Reynoso,E., Moffett,D., Goss,W.M., Dubner,G., Dickel,J., Reynolds,S., \& Giacani,E. 1997, ApJ, 491, 816

Reynoso,E., Velázquez,P., Dubner,G., \& Goss,W.M., 1999, AJ, 117, 1827.

Velázquez,P., Gómez,D., Dubner,G., Giménez de Castro,G., \& Costa,A., 1998, A\&A, 334, 1060.

Woltjer,L. 1972, ARA\&A, 10, 129 